\title{
Synthesis of 3-fluoropyrrolidines and 3-fluoroazetidines
}

\author{
Karel Piron, ${ }^{\$}$ Guido Verniest, ${ }^{\#}$ Eva Van Hende and Norbert De Kimpe* \\ Department of Sustainable Organic Chemistry and Technology, Faculty of Bioscience \\ Engineering, Ghent University, Coupure Links 653, B-9000 Ghent, Belgium \\ E-mail: norbert.dekimpe@UGent.be
}

Dedicated to Prof. Dr. Ferenc Fülöp on the occasion of his 60th birthday

DOI: $\underline{\text { http://dx.doi.org/10.3998/ark.5550190.0013.502 }}$

\begin{abstract}
A synthetic route towards 1-t-butoxycarbonyl-3-fluoro-3-methylpyrrolidine and 1-tbutoxycarbonyl-3-fluoro-3-methylazetidine, which are interesting building blocks for pharmaceutical compounds, is described. The key steps include a bromofluorination of appropriate alkenyl azides, followed by reduction to the corresponding amines and subsequent cyclization, yielding the 3-fluorinated azaheterocycles.
\end{abstract}

Keywords: Bromofluorination, 3-fluoropyrrolidines, 3-fluoroazetidines, alkenyl azides

\section{Introduction}

Site-selective fluorination has become an important strategy for developing new bioactive compounds, which is reflected by the numerous papers in the area of organofluorine chemistry in the last decade. ${ }^{1}$ The intensified research dealing with the synthesis of new fluorinated organic compounds has resulted in numerous new commercial applications in pharmaceutical chemistry and agrochemistry. ${ }^{2}$ More specifically, 3-fluoropyrrolidines and 3-fluoroazetidines are recognised as valuable building blocks in physiologically active compounds. 3Fluoropyrrolidines exhibit interesting biological activities, such as purine nucleoside phosphorylase (PNP) inhibitors ${ }^{3}$ and kinesin spindle protein (KSP) inhibitors, ${ }^{4}$ while 3fluoroazetidines are promising in the treatment of diabetes type $2^{5}$ and colon cancer. ${ }^{6}$

The main synthetic pathways so far toward these 3-monofluorinated pyrrolidines and azetidines dealt with the deoxofluorination of the corresponding 3-hydroxylated heterocycles using DAST (diethylaminosulfur trifluoride) or related deoxofluorinating agents, such as DeoxoFluor $^{\circledR}$ or morphDAST ${ }^{\circledR} .7$ Another strategy to obtain 3-fluoropyrrolidines made use of the 
electrophilic fluorinating reagent NFSI ( $N$-fluorodibenzenesulfonimide) to convert $\gamma$-lactams into the corresponding $\alpha$-fluorinated derivatives. Subsequent reduction of the carbonyl function then led to the desired 3-fluoropyrrolidines. ${ }^{8}$

Earlier research at our department demonstrated the use of $N$-(alkylidene or 1arylmethylidene)-2-propenylamines ${ }^{9}$ and $N$-(diphenylmethylidene)-2-propenylamines ${ }^{10}$ as suitable precursors for the synthesis of new 3-alkyl- and 3-aryl-3-fluoroazetidines. A regioselective bromofluorination of the carbon-carbon double bond of $N$-alkenylimines afforded the corresponding bromofluorinated $N$-alkylimines, which were subsequently treated with reducing agents to obtain the corresponding amines, yielding the desired 3 -fluoroazetidines after cyclization via intramolecular substitution of the bromine atom. In continuation of our interest in fluorinated azaheterocycles, ${ }^{11}$ we herein describe an alternative and optimized procedure for the synthesis of 1-t-butoxycarbonyl-3-fluoro-3-methylazetidine, and the application towards 1-tbutoxycarbonyl-3-fluoro-3-methylpyrrolidine, which are both of considerable pharmaceutical interest.

\section{Results and Discussion}

As described above, imines derived from benzaldehyde or benzophenone proved to be suitable protective groups for 2-propenylamines which readily undergo bromofluorination upon treatment with $\mathrm{N}$-bromosuccinimide (NBS) and triethylamine trishydrofluoride $\left(\mathrm{Et}_{3} \mathrm{~N} \cdot 3 \mathrm{HF}\right) .{ }^{12}$ The resulting bromofluorinated imines can be used to synthesize the corresponding 3-fluoroazetidines, as described above. ${ }^{9,10}$ At first, it was tried to extend this strategy toward the synthesis of 3-alkyl-3fluoropyrrolidines, using $N$-(diphenylmethylidene)-3-methyl-3-butenylamine $\mathbf{5}$ as starting material for the bromofluorination reaction (Scheme 1). The latter imine 5 was obtained via a 4step reaction sequence starting from commercially available 3-methyl-3-buten-1-ol 1. After tosylation of the hydroxyl function of 3-methyl-3-butenol and substitution of the tosylate by reaction with sodium azide, the azide function was reduced via a Staudinger reaction using triphenylphosphine in $\mathrm{Et}_{2} \mathrm{O} / \mathrm{H}_{2} \mathrm{O}(10: 1) .{ }^{13}$ Subsequently, the resulting 3-methyl-3-butenamine 4 was treated with benzophenone imine in dichloromethane to afford $\mathrm{N}$-(diphenylmethylidene)-3methyl-3-butenylamine 5, which was treated with $\mathrm{NBS}$ and $\mathrm{Et}_{3} \mathrm{~N} \cdot 3 \mathrm{HF}$ in order obtain $N$ (diphenylmethylidene)-4-bromo-3-fluoro-3-methylbutylamine 6. Unfortunately, this bromofluorination reaction proved unsuccessful and yielded mainly benzophenone due to iminium ion formation and hydrolysis during work-up. Attempts to isolate the bromofluorinated imine 6 directly from the reaction mixture without aqueous work-up, and subsequent use in following reactions failed. 

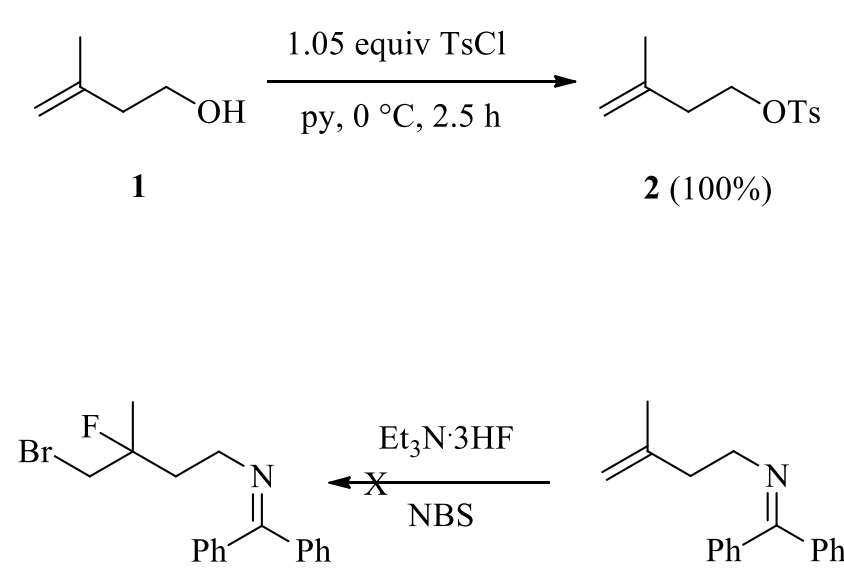

6

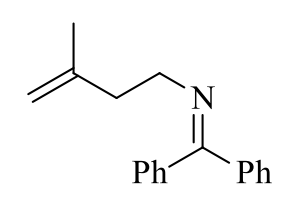

$5(96 \%)$

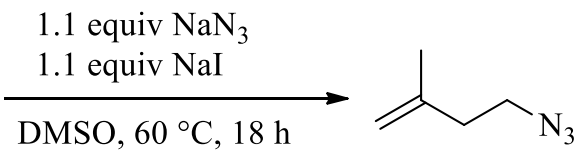

$3(81 \%)$

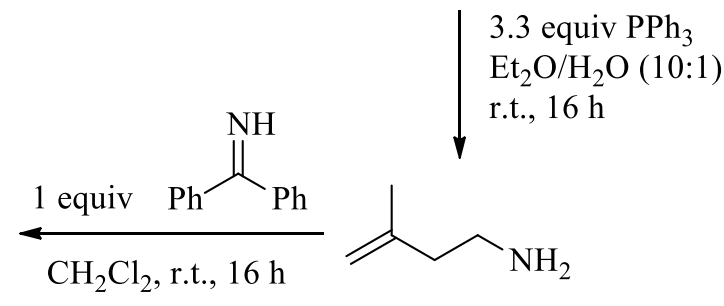

$4(68 \%)$

\section{Scheme 1}

Since the bromofluorination of $\mathrm{N}$-butenylimine $\mathbf{5}$ did not result in suitable precursors for the synthesis of fluoropyrrolidines, the bromofluorination reaction was evaluated using 4-azido-2methyl-1-butene $\mathbf{3}$ as substrate. The halogenation of alkenes in the presence of an azide functionality is known in literature. ${ }^{14}$ Indeed, 4-azido-2-methyl-1-butene 3 was very smoothly converted into the bromofluorinated analogue 7 by reaction with $\mathrm{Et}_{3} \mathrm{~N} \cdot 3 \mathrm{HF}$ and $\mathrm{NBS}$ in dichloromethane at $0{ }^{\circ} \mathrm{C}$ (Scheme 2). Although no problems have been observed in our hands, precautions should be taken into account to preclude the presence of residual sodium azide because treatment with $\mathrm{Et}_{3} \mathrm{~N} \cdot 3 \mathrm{HF}$ could give rise to formation of explosive $\mathrm{HN}_{3}$ (CAUTION). Therefore, the intermediate azide $\mathbf{3}$ should be washed thoroughly with water and purified prior to its use in following reactions. All reactions and isolation operations should be performed in a closed fume hood with a safety screen. The resulting 4-azido-1-bromo-2-fluoro-2-methylbutane 7, which was pure enough to use without purification, was subsequently treated with hydrogen gas ( 5 bar) over $\mathrm{Pd} / \mathrm{C}$ in the presence of $\mathrm{Boc}_{2} \mathrm{O}$, thereby trapping the resulting amine in situ affording the $N$-Boc amine $\mathbf{8}$ in good yield after chromatographic purification (87\%). In a final step toward the desired 3-fluoro-3-methylpyrrolidine 9, tert-butyl 4-bromo-3-fluoro-3methylbutylcarbamate $\mathbf{8}$ was treated with sodium hydride in DMF at room temperature for $1 \mathrm{~h}$, affording pure 3 -fluoropyrrolidine 9 in $73 \%$ yield. 


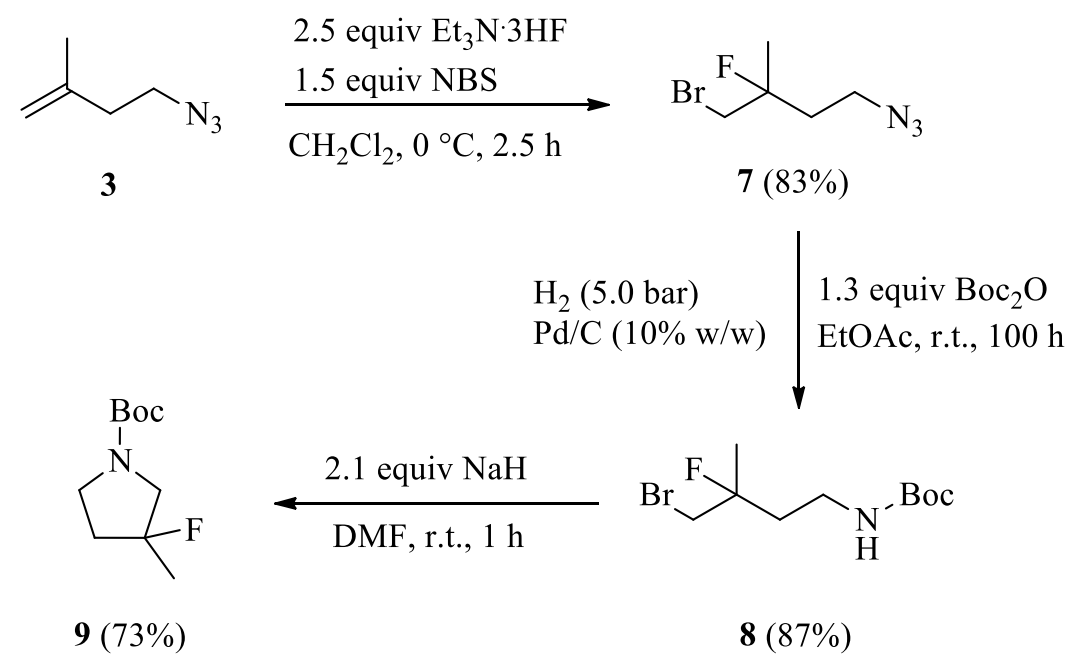

\section{Scheme 2}

$\mathrm{N}$-Boc-3-fluoro-3-methylpyrrolidine 9 was thus obtained via a 5-step reaction sequence with a good overall yield of 43\%, starting from cheap 3-methyl-3-buten-1-ol 1. This synthetic pathway is quite attractive since almost no side reactions occur, which avoids the need for chromatographic purification of the intermediates.

Having in hand an efficient method for the synthesis of 3 -fluoropyrrolidine 9, the above described methodology was extended toward the synthesis of 3-fluoroazetidine 14. Starting from 2-methyl-2-propenyl chloride 10, the envisaged 3-fluoro-3-methylazetidine 14 was obtained via a 4-step reaction sequence, applying analogous synthetic transformations as described above for the synthesis of fluoropyrrolidine 9 (Scheme 3). The reaction of 2-methyl-2-propenyl chloride $\mathbf{1 0}$ with sodium azide in DMSO at room temperature for $2.5 \mathrm{~h}$ gave the corresponding azide $\mathbf{1 1}^{15}$ in quantitative yield and the latter was used as substrate for the bromofluorination reaction using NBS and $\mathrm{Et}_{3} \mathrm{~N} \cdot 3 \mathrm{HF}$ (CAUTION: see above). Subsequent hydrogenation of the azide by treatment with hydrogen over $\mathrm{Pd} / \mathrm{C}$ in ethyl acetate in the presence of $\mathrm{Boc}_{2} \mathrm{O}$ resulted in the $\mathrm{N}$-Boc amine 13 in $29 \%$ yield after purification by flash chromatography. Deprotonation of the obtained $N$-Boc protected 3-bromo-2-fluoro-2-methylpropylamine 13 by sodium hydride in DMF gave rise to the envisaged 3-fluoroazetidine 14 in $76 \%$ yield. In the latter case, it is clear that the ring closing reaction after deprotonation takes considerably longer reaction times as compared to the formation of the pyrrolidine 9 from amine 8 (i.e. 23 hours reaction in case of azetidine 14, and 1 hour for the pyrrolidine 9). This is completely in line with the higher ring strain energy of azetidines compared to pyrrolidines. 


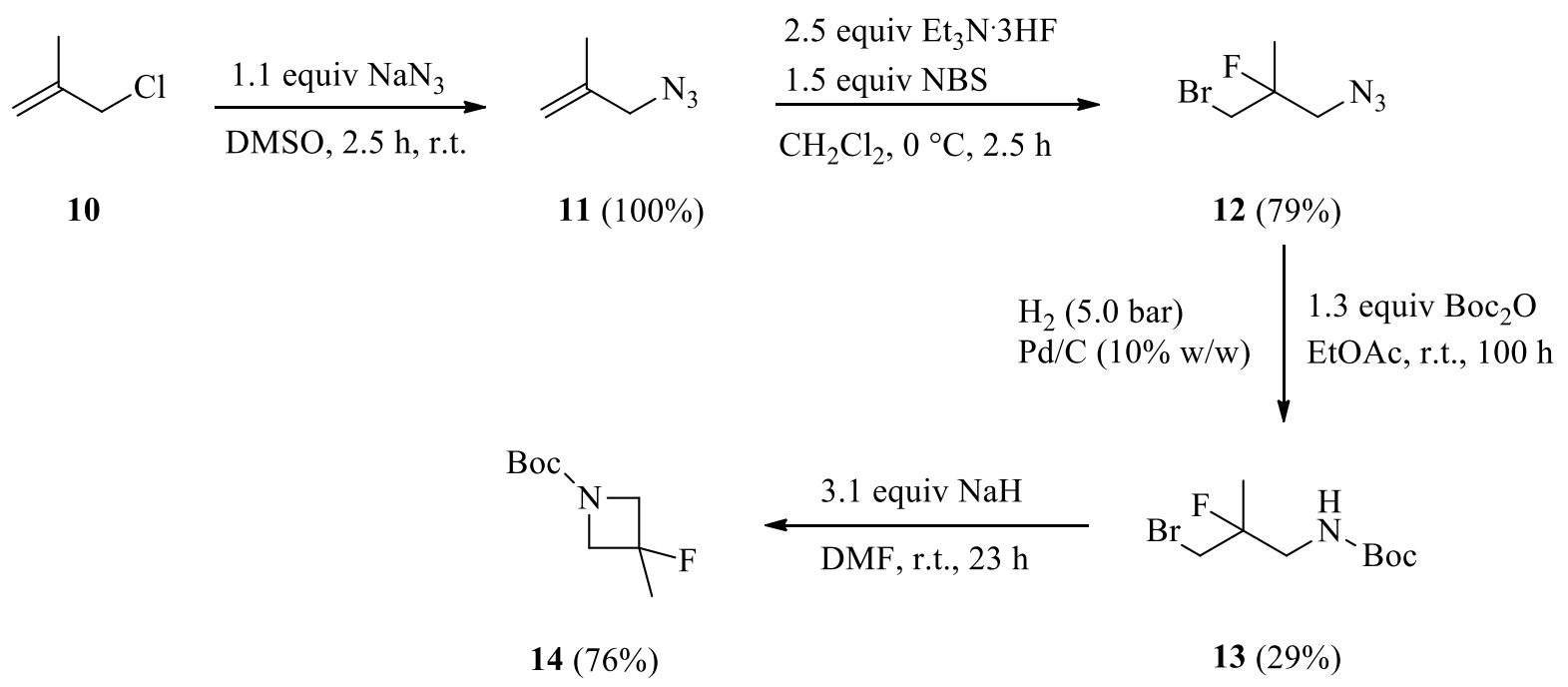

Scheme 3

\section{Conclusion}

In conclusion, a short and efficient route towards 1-t-butoxycarbonyl-3-fluoro-3-methylpyrrolidine was developed. The synthetic pathway included the bromofluorination of of 3-azido2-methylprop-1-ene, followed by reduction of the azide function and in situ trapping of the formed amine by $\mathrm{Boc}_{2} \mathrm{O}$, and finally ring closure via deprotonation of the carbamate $\mathrm{N}-\mathrm{H}$ using sodium hydride in DMF. This strategy was extended toward the synthesis of 1-Boc-3-fluoro-3methylazetidine. Both fluorinated heterocycles are useful building blocks in medicinal chemistry.

\section{Experimental Section}

General. ${ }^{1} \mathrm{H}$ NMR spectra were recorded on a Jeol Eclipse+ $300 \mathrm{NMR}$ spectrometer (300 MHz) with tetramethylsilane as internal standard. ${ }^{13} \mathrm{C}$ NMR spectra $(75 \mathrm{MHz})$ and ${ }^{19} \mathrm{~F}$ NMR spectra $(282 \mathrm{MHz})$ were also recorded with this spectrometer. IR spectra were measured with a Perkin Elmer Spectrum BX FT-IR spectrometer. Melting points were determined in open capillary tubes with a Büchi B-450 melting point apparatus. Electrospray (ES) mass spectra were recorded using a Agilent 1100 Series MS (4000V) mass spectrometer. Electron impact (EI) mass spectra were recorded using a HP 6890 GC coupled with a HP 5973 MSD (mass selective detector). Dichloromethane was dried over calcium hydride, other solvents were used as received from the supplier.

Synthesis of $\boldsymbol{N}$-(diphenylmethylidene)-3-methyl-3-butenylamine (5). To a solution of $1.36 \mathrm{~g}$ (16.0 mmol, 1 equiv) of 3-methyl-3-butenylamine 4 in dry $\mathrm{CH}_{2} \mathrm{Cl}_{2}(50 \mathrm{~mL})$ was added $2.90 \mathrm{~g}$ 
(16.0 mmol, 1 equiv) of benzophenone imine, and the resulting mixture was stirred for 16 hours at room temperature. After removal of the solvent in vacuo, $3.85 \mathrm{~g}$ (15.4 mmol, 96\% yield) of $\mathrm{N}$ (diphenylmethylidene)-3-methyl-3-butenylamine 5 was obtained as a yellow oil. ${ }^{1} \mathrm{H}$ NMR $\left(300 \mathrm{MHz}, \mathrm{CDCl}_{3}\right): \delta 1.65\left(3 \mathrm{H}, \mathrm{s}, \mathrm{CH}_{3}\right) ; 2.40\left(2 \mathrm{H}, \mathrm{t}, J=7.5 \mathrm{~Hz}, \underline{\mathrm{C}}_{2} \mathrm{CH}_{2} \mathrm{~N}\right) ; 3.51(2 \mathrm{H}, \mathrm{t}, J=7.5$ $\left.\mathrm{Hz}, \mathrm{CH}_{2} \mathrm{~N}\right) ; 4.67\left(1 \mathrm{H}, \mathrm{s}, \mathrm{C}=\mathrm{C}_{\mathrm{a}} \mathrm{H}_{\mathrm{b}}\right) ; 4.71\left(1 \mathrm{H}, \mathrm{s}, \mathrm{C}=\mathrm{CH}_{\mathrm{a}} \underline{\mathrm{H}_{\mathrm{b}}}\right) ; 7.16\left(2 \mathrm{H}, \mathrm{d}, J=7.8 \mathrm{~Hz}, 2\right.$ x $\left.\mathrm{CH}_{\mathrm{ar}}\right)$; 7.25-7.48 (6H, m, $\left.6 \times \mathrm{CH}_{\mathrm{ar}}\right) ; 7.59\left(2 \mathrm{H}, \mathrm{d}, J=7.8 \mathrm{~Hz}, 2 \times \mathrm{CH}_{\mathrm{ar}}\right) .{ }^{13} \mathrm{C} \mathrm{NMR}\left(75 \mathrm{MHz}, \mathrm{CDCl}_{3}\right): \delta$ $22.7\left(\mathrm{CH}_{3}\right) ; 39.3\left(\underline{\mathrm{CH}}_{2} \mathrm{CH}_{2} \mathrm{~N}\right) ; 52.5\left(\mathrm{CH}_{2} \mathrm{~N}\right) ; 111.1\left(\mathrm{C}=\underline{\mathrm{CH}}_{2}\right) ; 2$ x 127.8, 2 x 128,0, 2 x 128,3, 2 x 128,4 and $2 \times 129.8\left(10 \times \mathrm{CH}_{\mathrm{ar}}\right) ; 136.8\left(\mathrm{C}_{\mathrm{ar}, \mathrm{q}}\right) ; 140.0\left(\mathrm{C}_{\mathrm{ar}, \mathrm{q}}\right) ; 144.1\left(\underline{\mathrm{C}}=\mathrm{CH}_{2}\right) ; 168.2(\mathrm{C}=\mathrm{N})$. IR $\left(\mathrm{ATR}, \mathrm{cm}^{-1}\right): v=1622(\mathrm{C}=\mathrm{N}) ; 1598 ; 1446 ; 1362 ; 1195 ; 887 ; 693$. MS $(\mathrm{ES}+): \mathrm{m} / z(\%): 250$ $\left(\mathrm{M}+\mathrm{H}^{+}, 100\right)$.

Synthesis of 4-azido-1-bromo-2-fluoro-2-methylbutane (7). To a solution of $0.70 \mathrm{~g}$ (6.30 mmol, 1 equiv) of 3-methyl-3-butenylazide 3 in dry $\mathrm{CH}_{2} \mathrm{Cl}_{2}(10 \mathrm{~mL})$ at $0{ }^{\circ} \mathrm{C}$ was added $2.6 \mathrm{~mL}$ (15.75 mmol, 2.5 equiv) of $\mathrm{Et}_{3} \mathrm{~N} \cdot 3 \mathrm{HF}$ with a syringe under $\mathrm{N}_{2}$ atmosphere. After 5 minutes stirring, $1.68 \mathrm{~g}$ (9.45 mmol, 1.5 equiv) of NBS was added and the resulting mixture was stirred for 2.5 hours at $0{ }^{\circ} \mathrm{C}$ under nitrogen atmosphere. Subsequently, the mixture was poured in an aqueous solution of $1 \mathrm{M} \mathrm{NaOH}(20 \mathrm{~mL})$ and the water phase was extracted with $\mathrm{CH}_{2} \mathrm{Cl}_{2}(3 \times 10$ $\mathrm{mL})$. Then, the combined organic phases were washed with an aqueous solution of $1 \mathrm{M} \mathrm{NaOH} \mathrm{(2}$ x $10 \mathrm{~mL})$ and brine $(10 \mathrm{~mL})$. After drying $\left(\mathrm{MgSO}_{4}\right)$ and evaporation of the solvents in vacuo, $1,30 \mathrm{~g}$ of a red brown oil was obtained that contained mainly 4-azido-1-bromo-2-fluoro-2methylbutane 7 (purity of the obtained oil determined by GC analysis: 85\%). Approximate calculated yield of 7: $83 \%(5.26 \mathrm{mmol}, 1.10 \mathrm{~g}) .{ }^{1} \mathrm{H} \mathrm{NMR}\left(300 \mathrm{MHz}, \mathrm{CDCl}_{3}\right): \delta 1.52(3 \mathrm{H}, \mathrm{d}, J=$ $\left.21.5 \mathrm{~Hz}, \mathrm{CH}_{3}\right) ; 1.95-2.23\left(2 \mathrm{H}, \mathrm{m}, \mathrm{CH}_{2} \mathrm{CH}_{2} \mathrm{~N}_{3}\right) ; 3.45\left(2 \mathrm{H}, \mathrm{t}, J=7.2 \mathrm{~Hz}, \mathrm{CH}_{2} \mathrm{~N}_{3}\right) ; 3.47(2 \mathrm{H}, \mathrm{d}, J=$ $\left.14.9 \mathrm{~Hz}, \mathrm{CH}_{2} \mathrm{Br}\right) .{ }^{19} \mathrm{~F}$ NMR (282 MHz, $\left.\mathrm{CDCl}_{3}\right): \delta-145.7(1 \mathrm{~F}, \mathrm{~m}) .{ }^{13} \mathrm{C} \mathrm{NMR}\left(75 \mathrm{MHz}, \mathrm{CDCl}_{3}\right): \delta$ $23.6\left(\mathrm{~d}, J=24.3 \mathrm{~Hz}, \mathrm{CH}_{3}\right) ; 36.4\left(\mathrm{~d}, J=21.9 \mathrm{~Hz}, \underline{\mathrm{CH}}_{2} \mathrm{CH}_{2} \mathrm{~N}_{3}\right) ; 37.8\left(\mathrm{~d}, J=30.0 \mathrm{~Hz}, \mathrm{CH}_{2} \mathrm{Br}\right) ; 46.1$ $\left(\mathrm{d}, J=5.8 \mathrm{~Hz}, \mathrm{CH}_{2} \mathrm{~N}_{3}\right) ; 93.8(\mathrm{~d}, J=174.2 \mathrm{~Hz}, \mathrm{CF})$. IR (ATR, $\left.\mathrm{cm}^{-1}\right): v=2094\left(\mathrm{~N}_{3}\right) . \mathrm{MS}(\mathrm{ES}+)$ : $m / z(\%): 210 / 212\left(\mathrm{M}+\mathrm{H}^{+}, 100\right) ; 182 / 184\left(\mathrm{M}-\mathrm{N}_{2}+\mathrm{H}^{+}, 71\right)$.

Synthesis of tert-butyl 4-bromo-3-fluoro-3-methylbutylcarbamate (8). To a solution of 0.82 $\mathrm{g}$ of crude 4-azido-1-bromo-2-fluoro-2-methylbutane 7 (85\% purity; $3.32 \mathrm{mmol}, 1$ equiv) in EtOAc $(15 \mathrm{~mL})$ was added $0.94 \mathrm{~g}$ ( $4.31 \mathrm{mmol}, 1.3$ equiv) of $\mathrm{Boc}_{2} \mathrm{O}$ and $0.08 \mathrm{~g}$ of $\mathrm{Pd} / \mathrm{C}(10 \%$ $\mathrm{w} / \mathrm{w})$. The resulting mixture was stirred under $\mathrm{H}_{2}$ atmosphere $(5.0 \mathrm{bar}$ ) for 100 hours at room temperature. Then, the mixture was filtered through a pad of Celite ${ }^{\circledR}$ and the filtrate was concentrated in vacuo. Finally, the obtained crude product was purified via silica gel flash chromatography (hexane/EtOAc 9:1, $\left.\mathrm{R}_{\mathrm{f}}=0.14\right)$ to afford $0.82 \mathrm{~g}(2.89 \mathrm{mmol}, 87 \%$ yield) of tertbutyl 4-bromo-3-fluoro-3-methylbutylcarbamate 8 as a yellow oil. ${ }^{1} \mathrm{H} \mathrm{NMR}\left(300 \mathrm{MHz}, \mathrm{CDCl}_{3}\right)$ : $\delta 1.43\left(9 \mathrm{H}, \mathrm{s}, \mathrm{C}\left(\mathrm{CH}_{3}\right)_{3}\right) ; 1.50\left(3 \mathrm{H}, \mathrm{d}, J=22.0 \mathrm{~Hz}, \mathrm{CH}_{3} \mathrm{CF}\right) ; 1.86-2.12\left(2 \mathrm{H}, \mathrm{m}, \mathrm{C}_{2} \mathrm{CH}_{2} \mathrm{~N}\right) ; 3.18-$ $3.39\left(2 \mathrm{H}, \mathrm{m}, \mathrm{CH}_{2} \mathrm{~N}\right) ; 3.45\left(2 \mathrm{H}, \mathrm{d}, J=16.0 \mathrm{~Hz}, \mathrm{CH}_{2} \mathrm{Br}\right) ; 4.72(1 \mathrm{H}, \mathrm{s}(\mathrm{br}), \mathrm{NH}) .{ }^{19} \mathrm{~F} \mathrm{NMR}(282$ $\left.\mathrm{MHz}, \mathrm{CDCl}_{3}\right): \delta-145.5(1 \mathrm{~F}, \mathrm{~m}) .{ }^{13} \mathrm{C} \mathrm{NMR}\left(75 \mathrm{MHz}, \mathrm{CDCl}_{3}\right): \delta 23.3\left(\mathrm{~d}, J=23.0 \mathrm{~Hz}, \mathrm{CH}_{3} \mathrm{CF}\right)$; $28.4\left(\mathrm{C}\left(\underline{\mathrm{CH}}_{3}\right)_{3}\right) ; 35.7\left(\mathrm{CH}_{2} \underline{\mathrm{CH}}_{2} \mathrm{~N}\right) ; 37.5\left(\mathrm{~d}, J=20.7 \mathrm{~Hz}, \underline{\mathrm{CH}_{2}} \mathrm{CH}_{2} \mathrm{~N}\right) ; 38.1(\mathrm{~d}, J=28.9 \mathrm{~Hz}$, $\left.\mathrm{CH}_{2} \mathrm{Br}\right) ; 79.4\left(\underline{\mathrm{C}}\left(\mathrm{CH}_{3}\right)_{3}\right) ; 94.6(\mathrm{~d}, J=173.1 \mathrm{~Hz}, \mathrm{CF}) ; 155.8(\mathrm{C}=\mathrm{O}) . \mathrm{IR}\left(\mathrm{ATR}, \mathrm{cm}^{-1}\right): v=3352$ 
(NH); 1693 (C=O); 1167. GC-MS (EI): $m / z(\%): 226 / 228\left(\mathrm{M}^{+}-\mathrm{C}\left(\mathrm{CH}_{3}\right)_{3}, 2\right) ; 208 / 210(24) ; 128$ (20); 84 (10); 74 (18); 59 (44); $57\left(\mathrm{C}^{+}\left(\mathrm{CH}_{3}\right)_{3}, 100\right) ; 41$ (20).

Synthesis of tert-butyl 3-fluoro-3-methylpyrrolidine-1-carboxylate (9). To a suspension of $0.14 \mathrm{~g}$ (60\% in mineral oil, $3.55 \mathrm{mmol}, 2.1$ equiv) of $\mathrm{NaH}$ in DMF (10 mL) was added a solution of $0.48 \mathrm{~g}$ (1.69 mmol, 1 equiv) of tert-butyl 4-bromo-3-fluoro-3-methylbutylcarbamate 8 in DMF $(20 \mathrm{~mL})$ dropwise at $0{ }^{\circ} \mathrm{C}$. The resulting mixture was stirred for 1 hour at room temperature, after which the mixture was poured in ice-cold water $(30 \mathrm{~mL})$ and extracted with $\mathrm{Et}_{2} \mathrm{O}(3 \times 20 \mathrm{~mL})$. The combined organic phases were subsequently washed with brine $(3 \times 20$ $\mathrm{mL})$, dried $\left(\mathrm{MgSO}_{4}\right)$ and concentrated in vacuo. Afterwards, the mixture was dissolved in $\mathrm{CH}_{3} \mathrm{CN}(10 \mathrm{~mL})$ and the mineral oil was removed by extraction with heptane $(2 \times 5 \mathrm{~mL})$. After evaporation of the $\mathrm{CH}_{3} \mathrm{CN}$ in vacuo, $0.25 \mathrm{~g}$ (1.23 mmol, 73\% yield) of tert-butyl 3-fluoro-3methylpyrrolidine-1-carboxylate 9 was obtained as a yellow oil. $\left.{ }^{1} \mathrm{H} \mathrm{NMR} \mathrm{(300} \mathrm{MHz}, \mathrm{CDCl}_{3}\right): \delta$ $1.46\left(9 \mathrm{H}, \mathrm{s}, \mathrm{C}\left(\mathrm{CH}_{3}\right)_{3}\right) ; 1.53\left(3 \mathrm{H}, \mathrm{d}, J=19.8 \mathrm{~Hz}, \mathrm{CH}_{3} \mathrm{CF}\right) ; 1.73-2.22\left(2 \mathrm{H}, \mathrm{m}, \mathrm{NCH}_{2} \mathrm{CH}_{2}\right) ; 3.29$ $\left(1 \mathrm{H}, \mathrm{ddd}, J=34.7 \mathrm{~Hz}, 12.7 \mathrm{~Hz}, 7.2 \mathrm{~Hz}, \mathrm{NC}_{\underline{\mathrm{a}}} \mathrm{H}_{\mathrm{b}} \mathrm{CF}\right) ; 3.42-3.73\left(3 \mathrm{H}, \mathrm{m}, \mathrm{C}_{2} \mathrm{NCH}_{\mathrm{a}} \underline{\mathrm{H}_{b}} \mathrm{CF}\right) .{ }^{19} \mathrm{~F}$ NMR $\left(282 \mathrm{MHz}, \mathrm{CDCl}_{3}\right): \delta-142.9(1 \mathrm{~F}, \mathrm{~m}) .{ }^{13} \mathrm{C} \mathrm{NMR}\left(75 \mathrm{MHz}, \mathrm{CDCl}_{3}\right): \delta 21.8(\mathrm{~d}, J=25.3 \mathrm{~Hz}$, $\left.\underline{\mathrm{CH}}_{3} \mathrm{CF}\right) ; 28.4\left(\mathrm{C}\left(\underline{\mathrm{CH}}_{3}\right)_{3}\right) ; 36.8$ and $37.6\left(\mathrm{~d}, J=23.1 \mathrm{~Hz}, \mathrm{NCH}_{2} \underline{\mathrm{CH}}_{2}\right)^{*} ; 44.4$ and 44.8 $\left(\mathrm{NCH}_{2} \mathrm{CH}_{2}\right)^{*} ; 56.4$ and $56.8\left(\mathrm{~d}, J=24.8 \mathrm{~Hz}, \mathrm{NCH}_{2} \mathrm{CF}\right)^{*} ; 79.4\left(\underline{\mathrm{C}}\left(\mathrm{CH}_{3}\right)_{3}\right) ; 99.9$ and $100.7(\mathrm{~d}, J=$ $173.1 \mathrm{~Hz}, \mathrm{CF})^{*} ; 154.2(\mathrm{C}=\mathrm{O})$. IR (ATR, $\left.\mathrm{cm}^{-1}\right): v=1694(\mathrm{C}=\mathrm{O}) ; 1402 ; 1365 ; 1170 ; 1135 ; 1102$. GC-MS (EI): m/z (\%): $203\left(\mathrm{M}^{+}, 6\right) ; 148(31) ; 130\left(\mathrm{M}^{+}-\mathrm{OC}\left(\mathrm{CH}_{3}\right)_{3}, 49\right) ; 57\left(\mathrm{C}^{+}\left(\mathrm{CH}_{3}\right)_{3}, 100\right) ; 43$ (31); 41 (26). [ 'Different signals due to $N$-Boc rotamers].

Synthesis of 1-azido-3-bromo-2-fluoro-2-methylpropane (12). The synthetic procedure for compound 12 is analogous to the synthesis of 4-azido-1-bromo-2-fluoro-2-methylbutane 7. After bromofluorination of $1.00 \mathrm{~g}$ (10.30 mmol) of 3-azido-2-methylpropene 11 with NBS $(2.75 \mathrm{~g}$, $15.45 \mathrm{mmol}, 1.5$ equiv) and $\mathrm{Et}_{3} \mathrm{~N} \cdot 3 \mathrm{HF}$ (4.2 mL, $25.75 \mathrm{mmol}, 2.5$ equiv) in $\mathrm{CH}_{2} \mathrm{Cl}_{2}$ at $0^{\circ} \mathrm{C}$ for 2.5 $\mathrm{h}, 1.83 \mathrm{~g}$ of a red brown oil was obtained that contained mainly 1-azido-3-bromo-2-fluoro-2methylpropane 12 (purity of the obtained oil determined by GC analysis: 87\%). Approximate calculated yield of 12: $79 \%$ (8.12 mmol, $1.59 \mathrm{~g}){ }^{1} \mathrm{H}$ NMR (300 $\left.\mathrm{MHz}, \mathrm{CDCl}_{3}\right): \delta 1.53(3 \mathrm{H}, \mathrm{d}, J=$ $\left.20.9 \mathrm{~Hz}, \mathrm{CH}_{3}\right) ; 3.44-3.61$ (4H, m, $\left.\mathrm{CH}_{2} \mathrm{CFCH}_{2}\right) .{ }^{19} \mathrm{~F} \mathrm{NMR}\left(282 \mathrm{MHz}, \mathrm{CDCl}_{3}\right): \delta-149.3(1 \mathrm{~F}, \mathrm{~m})$. ${ }^{13} \mathrm{C} \mathrm{NMR}\left(75 \mathrm{MHz}, \mathrm{CDCl}_{3}\right): \delta 21.6\left(\mathrm{~d}, J=23.1 \mathrm{~Hz}, \mathrm{CH}_{3}\right) ; 34.7\left(\mathrm{~d}, J=30.0 \mathrm{~Hz}, \mathrm{CH}_{2} \mathrm{Br}\right) ; 56.0(\mathrm{~d}$, $\left.J=24.2 \mathrm{~Hz}, \mathrm{CH}_{2} \mathrm{~N}_{3}\right) ; 94.5(\mathrm{~d}, J=176.5 \mathrm{~Hz}, \mathrm{CF})$. IR (ATR, $\left.\mathrm{cm}^{-1}\right): v=2101\left(\mathrm{~N}_{3}\right)$. GC-MS (EI):

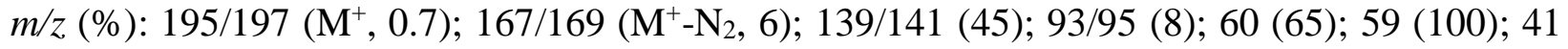
(22).

Synthesis of tert-butyl 3-bromo-2-fluoro-2-methylpropylcarbamate (13). The synthetic procedure for compound $\mathbf{1 3}$ is analogous to the synthesis of tert-butyl 4-bromo-3-fluoro-3methylbutylcarbamate 8. After hydrogenation of $0.70 \mathrm{~g}$ of crude 1-azido-3-bromo-2-fluoro-2methylpropane 12 (87\% purity, $3.11 \mathrm{mmol})$ using $0.07 \mathrm{~g}$ of $\mathrm{Pd} / \mathrm{C}(10 \% \mathrm{w} / \mathrm{w})$ under hydrogen atmosphere $\left(5.0\right.$ bar) in the presence of $\mathrm{Boc}_{2} \mathrm{O}(0.88 \mathrm{~g}, 4.04 \mathrm{mmol}, 1.3$ equiv) at room temperature for $100 \mathrm{~h}$ and purification via silica gel flash chromatography (hexane/EtOAc 19:1, $\left.\mathrm{R}_{\mathrm{f}}=0.10\right), \quad 0.24 \mathrm{~g} \quad(0.89 \mathrm{mmol}, \quad 29 \%$ yield $)$ of tert-butyl 3-bromo-2-fluoro-2methylpropylcarbamate $\mathbf{1 3}$ was obtained as a yellow oil. ${ }^{1} \mathrm{H} \mathrm{NMR}\left(300 \mathrm{MHz}, \mathrm{CDCl}_{3}\right): \delta 1.45$ 
$\left(9 \mathrm{H}, \mathrm{s}, \mathrm{C}\left(\mathrm{CH}_{3}\right)_{3}\right) ; 1.48\left(3 \mathrm{H}, \mathrm{d}, J=21.5 \mathrm{~Hz}, \mathrm{CH}_{3} \mathrm{CF}\right) ; 3.41-3.58\left(4 \mathrm{H}, \mathrm{m}, \mathrm{CH}_{2} \mathrm{CFCH}_{2}\right) ; 4.88(1 \mathrm{H}$, $\mathrm{s}(\mathrm{br}), \mathrm{NH}) .{ }^{19} \mathrm{~F}$ NMR $\left(282 \mathrm{MHz}, \mathrm{CDCl}_{3}\right): \delta-151.7(1 \mathrm{~F}, \mathrm{~m}) .{ }^{13} \mathrm{C} \mathrm{NMR}\left(75 \mathrm{MHz}, \mathrm{CDCl}_{3}\right): \delta 21.3$ $\left(\mathrm{d}, J=23.1 \mathrm{~Hz}, \underline{\mathrm{C}} \mathrm{H}_{3} \mathrm{CF}\right) ; 28.2\left(\mathrm{C}\left(\underline{\mathrm{CH}}_{3}\right)_{3}\right) ; 35.9$ (d, $\left.J=27.7 \mathrm{~Hz}, \mathrm{CH}_{2} \mathrm{Br}\right) ; 46.4(\mathrm{~d}, J=23.1 \mathrm{~Hz}$, $\left.\mathrm{CH}_{2} \mathrm{~N}\right) ; 79.4\left(\underline{\mathrm{C}}\left(\mathrm{CH}_{3}\right)_{3}\right) ; 94.6(\mathrm{~d}, J=175.3 \mathrm{~Hz}, \mathrm{CF}) ; 155.9(\mathrm{C}=\mathrm{O}) . \mathrm{IR}\left(\mathrm{ATR}, \mathrm{cm}^{-1}\right): v=3349$ $(\mathrm{NH}) ; 1697(\mathrm{C}=\mathrm{O}) ; 1161$. GC-MS (EI): $m / z(\%): 254 / 256\left(\mathrm{M}^{+}-\mathrm{CH}_{3}, 0.2\right) ; 194 / 196$ (3); 130 (46); 59 (56); $57\left(\mathrm{C}^{+}\left(\mathrm{CH}_{3}\right)_{3}, 100\right) ; 41(21)$.

Synthesis of tert-butyl 3-fluoro-3-methylazetidine-1-carboxylate (14). The synthetic procedure for compound $\mathbf{1 4}$ is analogous to the synthesis of tert-butyl 3-fluoro-3methylpyrrolidine-1-carboxylate 9 . After reaction of $0.15 \mathrm{~g}(0.55 \mathrm{mmol})$ of tert-butyl 3-bromo-2fluoro-2-methylpropylcarbamate 13 with $\mathrm{NaH}$ (69 mg of a $60 \%$ dispersion of $\mathrm{NaH}$ in mineral oil, $1.72 \mathrm{mmol}, 3.1$ equiv) in $5 \mathrm{~mL}$ of DMF at room temperature for $23 \mathrm{~h}, 80 \mathrm{mg}(0.423 \mathrm{mmol}, 76 \%$ yield) of tert-butyl 3-fluoro-3-methylazetidine-1-carboxylate 14 was obtained as a yellow oil. ${ }^{1} \mathrm{H}$ NMR (300 MHz, $\left.\mathrm{CDCl}_{3}\right): \delta 1.45\left(9 \mathrm{H}, \mathrm{s}, \mathrm{C}\left(\mathrm{CH}_{3}\right)_{3}\right) ; 1.60\left(3 \mathrm{H}, \mathrm{d}, J=21.5 \mathrm{~Hz}, \mathrm{CH}_{3} \mathrm{CF}\right) ; 3.87(2 \mathrm{H}$, ddd, $\left.J=17.3 \mathrm{~Hz}, 9.9 \mathrm{~Hz}, 1.3 \mathrm{~Hz},\left(\mathrm{C}_{\underline{\mathrm{a}}} \mathrm{H}_{\mathrm{b}}\right)_{2} \mathrm{~N}\right)$; $4.09\left(2 \mathrm{H}, \mathrm{dd}, J=21.0 \mathrm{~Hz}, 9.9 \mathrm{~Hz},\left(\mathrm{CH}_{\mathrm{a}} \underline{\mathrm{H}_{\mathrm{b}}}\right)_{2} \mathrm{~N}\right)$. ${ }^{19} \mathrm{~F}$ NMR (282 MHz, $\left.\mathrm{CDCl}_{3}\right): \delta-142.1(1 \mathrm{~F}, \mathrm{~m}) .{ }^{13} \mathrm{C} \mathrm{NMR}\left(75 \mathrm{MHz}, \mathrm{CDCl}_{3}\right): \delta 22.9(\mathrm{~d}, J=26.6$ $\left.\mathrm{Hz}, \underline{\mathrm{C}} \mathrm{H}_{3} \mathrm{CF}\right) ; 28.3\left(\mathrm{C}\left(\underline{\mathrm{CH}}_{3}\right)_{3}\right) ; 61.1\left(\mathrm{~d}, J=23.1 \mathrm{~Hz}, \mathrm{CH}_{2} \mathrm{NCH}_{2}\right) ; 79.9\left(\underline{\mathrm{C}}\left(\mathrm{CH}_{3}\right)_{3}\right) ; 89.7(\mathrm{~d}, J=$ $203.1 \mathrm{~Hz}, \mathrm{CF}) ; 156.3(\mathrm{C}=\mathrm{O})$. IR (ATR, $\left.\mathrm{cm}^{-1}\right): v=1707(\mathrm{C}=\mathrm{O}) ; 1392 ; 1366 ; 1175 ; 1106 ; 1146$. GC-MS (EI): $m / z(\%): 189\left(\mathrm{M}^{+}, 0.9\right) ; 174\left(\mathrm{M}^{+}-\mathrm{CH}_{3}, 2\right) ; 134(13) ; 116\left(\mathrm{M}^{+}-\mathrm{OC}\left(\mathrm{CH}_{3}\right)_{3}, 33\right) ; 89$ (15); $57\left(\mathrm{C}^{+}\left(\mathrm{CH}_{3}\right)_{3}, 100\right) ; 41(25)$.

\section{Acknowledgements}

The authors are indebted to the Research Foundation - Flanders (FWO-Flanders), Ghent University (GOA, BOF) Janssen Research and Development, a Division of Janssen Pharmaceutica NV, for financial support.

\section{References}

${ }^{\$}$ Aspirant of the Research Foundation - Flanders (FWO - Vlaanderen).

\#Postdoctoral Fellow of the Research Foundation - Flanders (FWO - Vlaanderen). Current address: Department of Chemistry, Faculty of Sciences and Bio-engineering Sciences, Vrije Universiteit Brussel, Pleinlaan 2, B-1050 Brussels, Belgium.

1. (a) Muller, K.; Faeh, C.; Diederich, F. Science 2007, 317, 1881. (b) O’Hagan, D. Chem. Soc. Rev. 2008, 37, 308. (c) Purser, S.; Moore, P. R.; Swallow, S.; Gouverneur, V. Chem. Soc. Rev. 2008, 37, 320. (d) Kirk, K. L. Org. Process Res. Dev. 2008, 12, 305. (e) Hagmann, W. K. J. Med. Chem. 2008, 51, 4359.

2. For examples, see: (a) Papeo, G. M. E.; Caronni, D.; Dalvit, C.; Giordano, P.; Mongelli, N.; Veronesi, M.; Ciprandi, F. Eur. Pat. Appl. 2008, EP 1923397. (b) Grabstein, K. H.; Wang, 
A.; Nairn, N.; Winblade; G.; Thomas, J. U.S. Pat. Appl. Publ. 2008, US 2008096819. (c) Edmondson, S. D.; Mastracchio, A.; Mathvink, R. J.; He, J.; Harper, B.; Park, Y.- J.; Beconi, M.; Di Salvo, J.; Eiermann, G. J.; He, H.; Leiting, B.; Leone, J. F.; Levorse, D. A.; Lyons, K.; Patel, R. A.; Patel, S. B.; Petrov, A.; Scapin, G.; Shang, J.; Roy, R. S.; Smith, A.; Wu, J. K.; Xu, S.; Zhu, B.; Thornberry, N. A.; Weber, A. E. J. Med. Chem. 2006, 49, 3614. (d) Celanire, S.; Quere, L.; Denonne, F.; Provins, L. PCT Int. Appl. 2007, WO 2007048595. (e) Keith, J. M.; Gomez, L. A.; Letavic, M. A.; Ly, L. S.; Jablonowski, J. A.; Seierstad, M.; Barbier, A. J.; Wilson, S. J.; Boggs, J. D.; Fraser, I. C.; Mazur, C.; Lovenberg, T. W.; Carruthers, N. I. Bioorg. Med. Chem. Lett. 2007, 17, 702. (f) Parker, J. C.; Hulin, B. US Pat. Appl. Publ. 2005, US 2005043292.

3. Mason, J. M.; Murkin, A. S.; Li, L.; Schramm, V. L.; Gainsford, G. J.; Skelton, B. W. J. Med. Chem. 2008, 51, 5880.

4. Barsanti, P. A.; Xia, Y.; Wang, W.; Mendenhall, K. G.; Lagniton, L. M.; Ramurthy, S.; Phillips, M. C.; Subramanian, S.; Boyce, R.; Brammeier, N. M.; Constantine, R.; Duhl, D.; Walter, A. O.; Abrams, T. J.; Renhowe, P. A. US Pat. Appl. Publ. 2007, US 20070037853.

5. (a) Hulin, B.; Parker, J.C. PCT Int. Appl, 2003, WO 2003101958. (b) Ferraris, D.; Belyakov, S.; Li, W.; Oliver, E.; Ko, Y.; Calvin, D.; Lautar, S.; Thomas, B.; Rojas, C. Curr. Top. Med. Chem. 2007, 7, 597.

6. Aay, N.; Anand, N. K.; Bowles, O. J.; Bussenius, J.; Costanzo, S.; Curtis, J. K.; Dubenko, L.; Joshi, A. A.; Kennedy, A. R.; Kim, A. I.; Koltun, E.; Manalo, J. L.; Peto, C. J.; Rice, K. D.; Tsang, T. H. PCT Int. Appl. 2007, WO 200704451.

7. For examples, see: (a) Demange, L.; Cluzeau, J.; Menez, A.; Dugave, C. Tetrahedron Lett. 2001, 42, 651. (b) Doi, M.; Nishi, Y.; Kiritoshi, N.; Iwata, T.; Nago, M.; Nakano, H.; Uchiyama, S.; Nakazawa, T.; Wakamiya, T.; Kobayashi, Y. Tetrahedron 2002, 58, 8453. (c) Yang, W.; Wang, Y.; Roberge, J. Y.; Ma, Z.; Liu, Y.; Lawrence, R. M.; Rotella, D. P.; Seethala, R.; Feyen, J. H. M.; Dickson, J. K. Jr. Bioorg. Med. Chem. Lett. 2005, 15, 1225. (d) Xu, J.; Wei, L.; Mastracchio, A.; Edmondson, S. D. PCT Int. Appl. 2005, WO 2005108382. (e) Renslo, A. R.; Atuegbu, A.; Herradura, P.; Jaishankar, P.; Ji, M.; Leach, K. L.; Huband, M. D.; Dermyer, M. R.; Wu, L.; Vara Prasad, J. V. N.; Gordeev M. F. Bioorg. Med. Chem. Lett. 2007, 17, 5036.

8. (a) Inagaki, H.; Miyauchi, S.; Miyauchi, R. N.; Kawato, H.C.; Ohki, H.; Matsuhashi, N.; Kawakami, K.; Takahashi, H.; Takemura, M. J. Med. Chem. 2003, 46, 1005. (b) Suzuki, T.; Goto, T.; Hamashima, Y.; Sodeoka, M. J. Org. Chem. 2007, 72, 246.

9. Van Brabandt, W.; Verniest, G.; De Smaele, D.; Duvey, G.; De Kimpe, N. J. Org. Chem. 2006, 71, 7100.

10. Van Hende, E.; Verniest, G.; Deroose, F.; Thuring, J. W.; Macdonald, G.; De Kimpe, N. J. Org. Chem. 2009, 74, 2250.

11. For recent examples, see: (a) Surmont, R.; Verniest, G.; Thuring, J. W.; ten Holte, P. Deroose, F.; De Kimpe, N. Org. Biomol. Chem. 2010, 8, 4514. (b) Verniest, G.; Piron, K.; Van Hende, E.; Thuring, J. W.; Macdonald, G.; Deroose, F.; De Kimpe, N. Org. Biomol. 
Chem. 2010, 8, 2509. (c) Surmont, R.; Verniest, G.; Thuring, J. W.; Macdonald, G.; Deroose, F.; De Kimpe, N. J. Org. Chem. 2010, 75, 929. (d) Surmont, R.; Verniest, G.; Deweweire, A.; Thuring, J. W.; Macdonald, G.; Deroose, F.; De Kimpe, N. Synlett 2009, 12, 1933. (e) Van Hende, E.; Verniest, G.; Thuring, J. W.; Macdonald, G.; Deroose, F.; De Kimpe, N. Synlett 2009, 11, 1765. (f) Verniest, G.; Surmont, R.; Van Hende, E.; Deweweire, A. De Kimpe, N. J. Org. Chem. 2008, 73, 5458.

12. Alvernhe, G.; Laurent, A.; Haufe, G. Synthesis 1987, 6, 562.

13. (a) Vaultier, M.; Knouzi, N.; Carrie, R. Tetrahedron Lett. 1983, 24, 763. (b) Leffler, J. E.; Temple, R. D. J. Am. Chem. Soc. 1967, 89, 5235. (c) Belmant, C.; Nury, P. PCT Int. Appl. 2005, WO 2005054258.

14. (a) Saito, H.; Suzuki, F.; Hirata, T. Chem Pharm. Bull. 1989, 37, 2298. (b) Basso, A.; Banfi, L.; Riva, R.; Guanti, G. J. Org. Chem. 2005, 70, 575.

15. Arimoto, M.; Yamaguchi, H.; Fujita, E.; Ochiai, M.; Nagao, Y. Tetrahedron Lett. 1987, 28, 6289. 